\title{
A Supplemental Technique for Preoperative Evaluation of Giant Intracranial Aneurysm
}

\author{
Hua-wei Wang ${ }^{1, *}$ Chen Wu ${ }^{1, *}$ Zhe Xue ${ }^{1, *}$ Xu-jun Shu ${ }^{1} \quad$ Zheng-hui Sun ${ }^{1}$ \\ ${ }^{1}$ Chinese PLA General Hospital, Neurosurgery, Beijing, China \\ Address for correspondence Zhenghui Sun, MD, Chinese PLA General \\ J Neurol Surg A Cent Eur Neurosurg 2021;82:424-429. \\ Hospital, Neurosurgery, Beijing, China (e-mail: szh_301@126.com).
}

\begin{abstract}
Background Preoperative planning mainly relies on digital subtraction angiography (DSA) and computed tomography angiography. However, neither technique can reveal thrombi in giant intracranial aneurysms (GIAs). In this study, we aimed to reconstruct the circulating and noncirculating parts of GIAs with the time-of-flight (TOF) and motion-sensitized driven-equilibrium (MSDE) sequences with 3D Slicer to reveal an integrated presentation of GIAs, compare its accuracy, and validate the usefulness for preoperative planning.

Material and Methods Patients with GIAs who were treated with microsurgery in our department were included in this study. Both the TOF and MSDE sequence data for each patient were loaded into 3D Slicer for reconstruction and segmentation. The parameters measured by 3D Slicer were compared with those measured by DSA.

Results The mean diameter for all GIAs was $28.7 \pm 1.5 \mathrm{~mm}$ (range, 25.9-31.9 mm). The mean diameter for all GIAs measured by DSA and 3D Slicer was $24.46 \pm 5.25$ and $28.66 \pm 1.48 \mathrm{~mm}$, respectively $(t=4.948, p<0.01)$. When only the nonthrombotic GIAs were included, the mean diameter measured by DSA and 3D Slicer was

\section{Keywords}

- intracranial aneurysm

- microsurgery

- magnetic resonance imaging

- software $28.69 \pm 2.03$ and $28.97 \pm 1.79 \mathrm{~mm}$, respectively $(t=1.023, p=0.323)$. The mean aneurysmal volume was $8,292.6 \pm 1,175.1 \mathrm{~mm}^{3}$ and the mean thrombotic volume was $3,590.0 \pm 1,003.7 \mathrm{~mm}^{3}$.

Conclusion The MSDE sequence brings diagnostic benefits as a comparison to other MRI sequences. Reconstruction of GIAs with 3D Slicer is a low-cost, dependable, and useful supplemental technique for surgical planning.
\end{abstract}

\section{Introduction}

Giant intracranial aneurysms (GIAs) are defined as aneurysms of $>25 \mathrm{~mm}$ in diameter and constitute 2.5 to $5.0 \%$ of all intracranial aneurysms. ${ }^{1-3}$ GIAs are more tumorlike than small aneurysms. The most common clinical presentation of a GIA is a local mass effect resulting in cranial nerve palsy, visual field defects, or limb weakness. ${ }^{2,4}$ Endovascular treatment for GIAs is limited due to its failure to relieve such mass effects, high cost for more coils, and high complication rate. ${ }^{5}$

${ }^{*}$ These authors contributed equally to this manuscript and should be considered as the co-first authors.

Thus, in certain cases, open surgery may be the best choice. However, GIAs have always been one of the most difficult cerebrovascular lesions to surgically manage. Despite the advances in microsurgical techniques, the corresponding surgical morbidity and mortality rates have remained as high as $20 \%{ }^{5,6}$ Therefore, meticulous imaging studies and individualized planning are required before surgery.

In the clinical setting, preoperative planning mainly involves digital subtraction angiography (DSA) and computed tomography angiography (CTA), which can always provide precise information about the circulating parts of GIAs and their patent arteries. However, neither examination technique can reveal the noncirculating parts of GIAs, such as thrombi. Spontaneous

(c) 2021. Thieme. All rights reserved. Georg Thieme Verlag KG,

Rüdigerstraße 14,

70469 Stuttgart, Germany
DOI https://doi.org/ 10.1055/s-0040-1721006. ISSN 2193-6315. 
thrombotic aneurysms can constitute up to $60 \%$ of all GIAs ${ }^{3,7}$; thus, the diameters and real morphology of GIAs are often likely to be underestimated by DSA or CTA. ${ }^{8}$ Additionally, because they behave like tumors, GIAs can compress the surrounding parenchyma and shift the brain structures from their normal locations. These changes also cannot be recognized only by DSA or CTA; therefore, other techniques are required to provide more information about GIAs for preoperative planning.

The utility of magnetic resonance imaging (MRI), a useful noninvasive examination technique, has been increasing in the evaluation of GIAs. ${ }^{8}$ The time-of-flight (TOF) sequence, mainly referred to as magnetic resonance angiography (MRA), has an ability to visualize circulating parts of aneurysms similar to the abilities of DSA and CTA. ${ }^{9,10}$ Motionsensitized driven equilibrium (MSDE) is another established sequence that can distinguish GIA thrombi from artifacts, ${ }^{11,12}$ many of which are caused by turbulent blood flow. ${ }^{8,13}$ 3D Slicer (http://www.slicer.org; - Fig. 1) is a free software for medical image visualization and analysis. This software supports image segmentation, registration, and multimodal visualization. In the present study, we reconstructed the circulating and noncirculating parts of GIAs with TOF and MSDE sequences by 3D Slicer (Version 4.6.2 for Mac OS X) to reveal an integrated presentation of GIAs and validate its usefulness for preoperative planning.

\section{Material and Methods}

\section{Patients}

After being approved by the institutional ethics committee of Chinese PLA general hospital, we retrospectively reviewed patients with GIAs who were treated surgically in our department between July 2015 and July 2018. Only the patients with preoperative TOF and MSDE MRI sequence and DSA imaging were included. Thrombi were also counted as parts of GIAs when measuring diameters. The maximum diameters were determined by both DSA and MRI. The exclusion criteria were (1) ruptured aneurysms, regardless of whether the GIAs were responsible for the hemorrhage and (2) previous treatment of the GIAs by microsurgery or endovascular intervention. At last, 31 consecutive patients with 31 GIAs were included in the study.

\section{Segmentation and Measurement}

Both the TOF and MSDE sequence data for every case were loaded into 3D Slicer, which was installed in a personal computer (MacBook Pro, 2.6 GHz Intel Core i5; Apple Inc., Cupertino, California, United States). GIA reconstructions were independently performed by two neurosurgeons who received the same guided training on the reconstruction procedure until they attained adequate proficiency. The reconstructions were
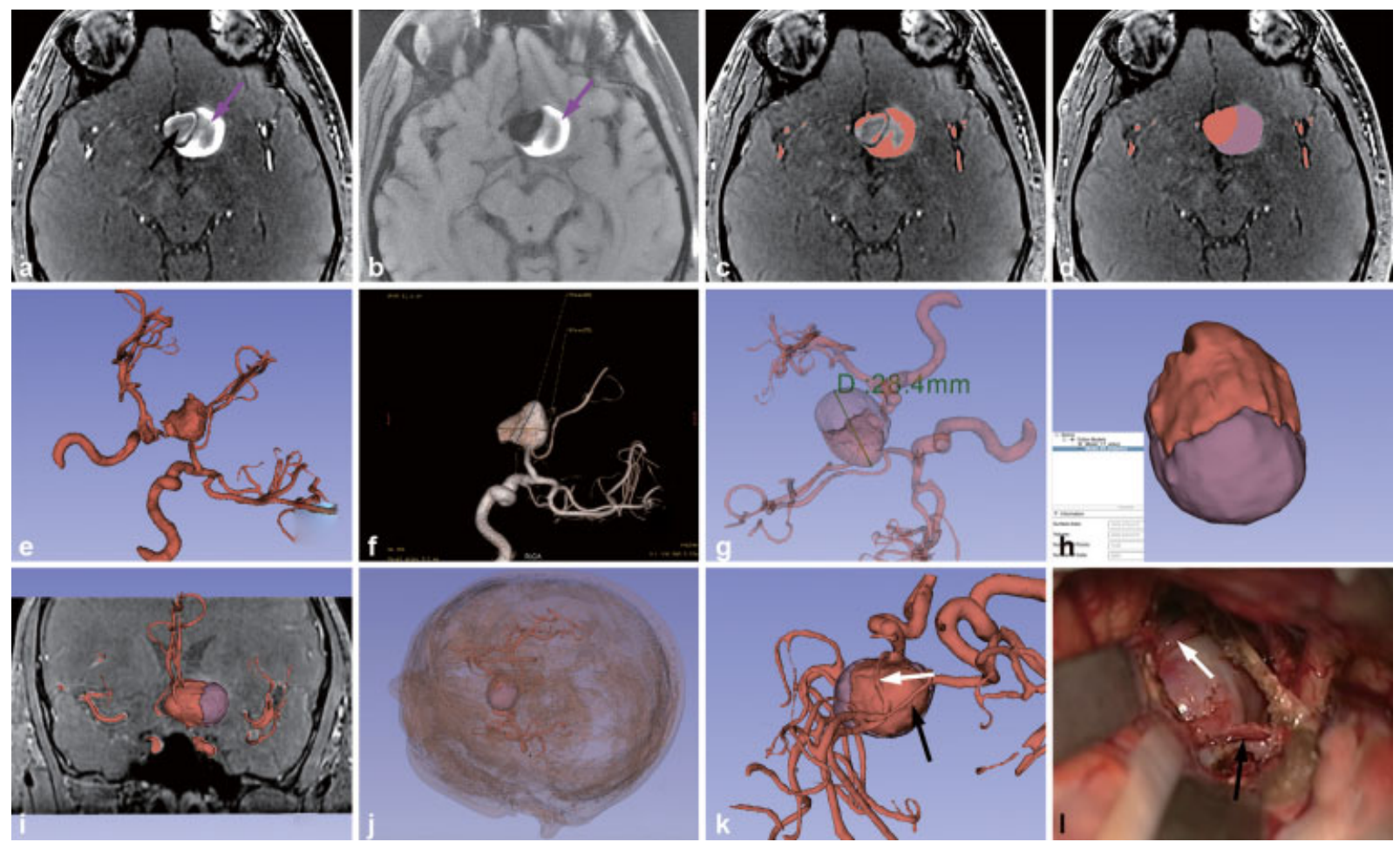

Fig. 1 The (a) time of flight (TOF) and (b) motion-sensitized driven-equilibrium (MSDE) sequence data were imported into 3D Slicer in Digital Imaging and Communications in Medicine (DICOM) format. Three compositions were identified after comparison at the same anatomical slides between the two sequences: blood flow, thrombi (purple arrows), and artifacts (black arrow). (c) The giant intracranial aneurysm (GIA) was first automatically marked with the Threshold Effect tool. (d) Next, the Paint Effect tool was used to manually prune and modify the structures. Finally, (e) the segmentation was converted to a 3D model and (f) this was similar to digital subtraction angiography (DSA) at the same angle. Integration of the thrombotic and circulatory parts together produced a whole model of the GIA and allowed for extraction of its ( $g$ ) diameter and (h) volume. The reconstructed GIA was integrated (i) with slide images and (j) into a head model to obtain more information. (k) The GIA model could be rotated at any angle, and two arteries (black and white arrows) could be verified in the surgical view angle. (I) The arteries (black and white arrows) could be confirmed during surgery in the same vi. 

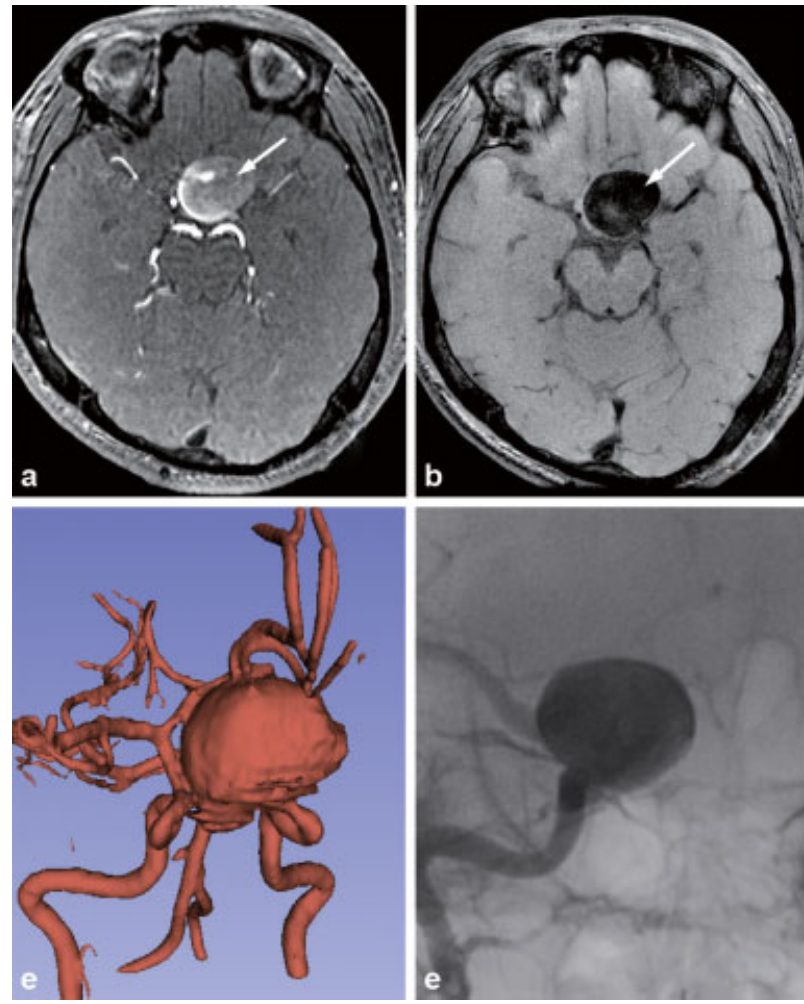
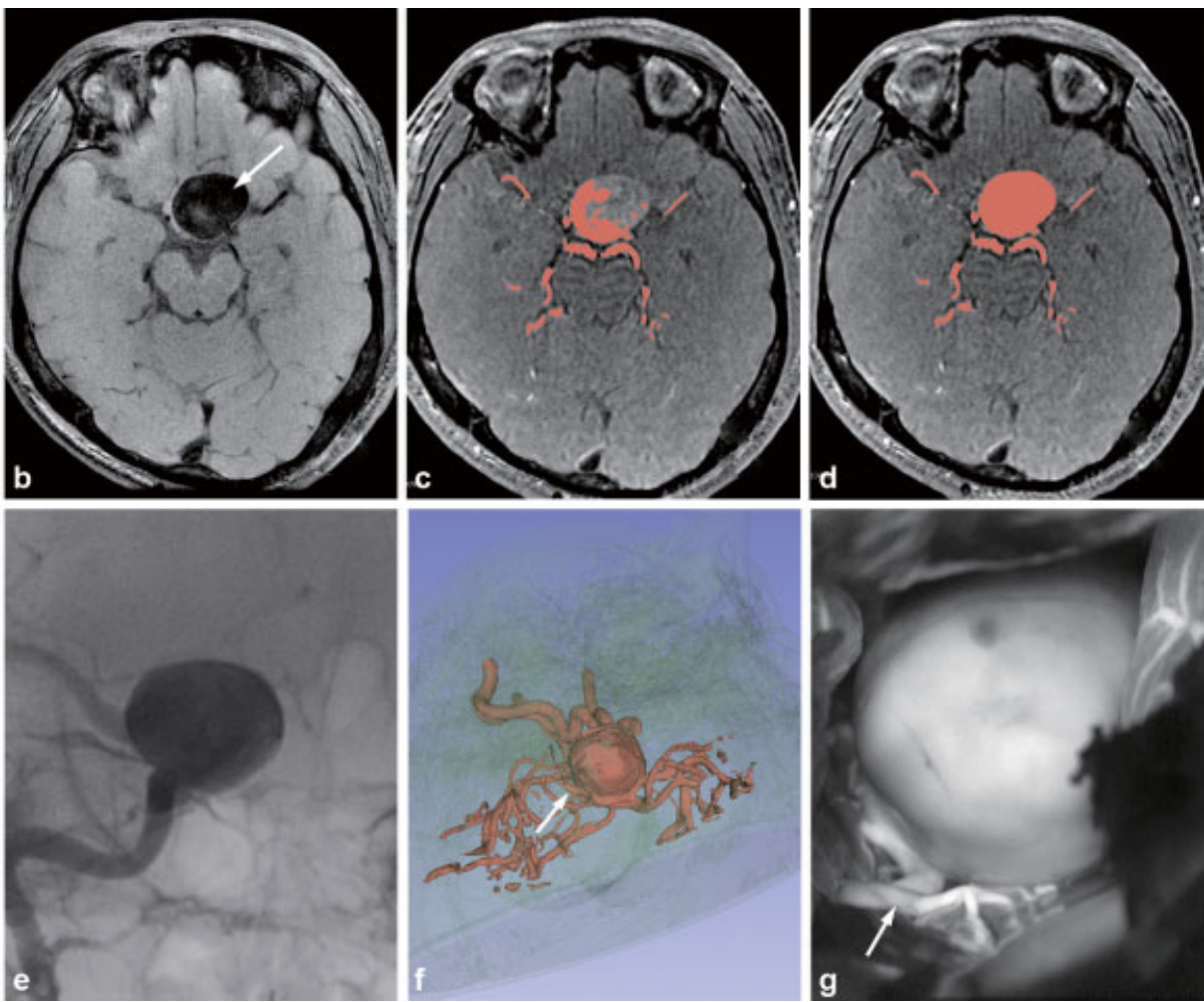

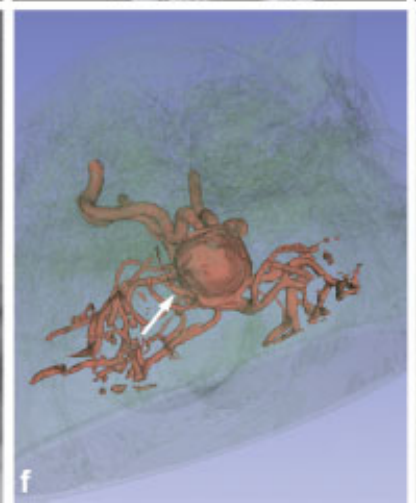

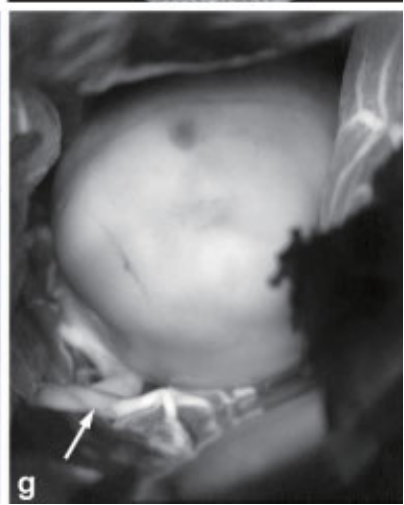

Fig. 2 Magnetic resonance imaging (MRI) examination of (a) time of flight (TOF) and (b) motion-sensitized driven-equilibrium (MSDE) sequences confirmed a nonthrombotic giant intracranial aneurysm (GIA) affected by artifacts (arrows). (c) First, the GIA was automatically marked with the Threshold Effect tool. (d) We then manually pruned and painted the whole aneurysm with Structure 17 using the Paint Effect tool. (e) Finally, the segmentation was converted to a 3D model with a smooth contour. (f) The morphology on 2D digital subtraction angiography (DSA) also confirmed the nonthrombotic GIA. (g) We then integrated the model into the reconstructed skull with semitransparency and rotated it to the neurosurgeon's view. The view could be adjusted to any angle with the computer mouse. Both the (g) reconstructed model and $(h)$ intraoperative fluorescence contrast image confirmed a surrounding perforator artery (arrows), proving the accuracy and usefulness of the reconstruction by 3D Slicer for preoperative planning.

mainly based on TOF sequences. Three compositions were identified in the TOF sequences: blood flow, thrombi, and artifacts. The MSDE sequences were used as a reference for distinguishing thrombi from artifacts in the TOF sequences.

The Threshold Effect tool was applied to automatically mark the circulatory parts of the GIAs and the cerebrovascular circulation (-Figs. 1 and 2). Manual drawing was then performed with the Paint Effect tool to prune or modify the colored structures slice by slice. All of the manual procedures covered $\sim 25$ to 50 slices according to the range of thrombi or artifacts in the GIAs. Finally, the resulting segmentation was converted to a 3D model and rendered with a smooth contour by the Merge and Build tool. Information on the volume of the thrombi and aneurysms could be instantly obtained from the model. The maximum diameters were measured by the two points that were farthest apart from each other within the reconstructed GIAs. Both values measured by the two independent neurosurgeons were recorded, and the average was calculated as the final result. The time required for every reconstruction was recorded.

For the 3D DSA, images were reconstructed by virtual reality on an Advantage Workstation (GE Healthcare). DSA data were stored in the workstation, and the GIA parameters were measured by a neuroradiologist who did not participate in the parameter measurement by 3D Slicer.

\section{Statistical Analysis}

After confirmation of the data distribution, the diameters of the GIAs were determined (median or mean \pm standard deviation), and a paired $t$-test was used for comparison between DSA and 3D reconstruction. To test for interobserver agreement, the intraclass correlation coefficient (ICC) for DSA and 3D reconstruction was calculated using a one- and two-way random-effects model, respectively, and was used as evidence of reconstructive stability. The coincidence rate for a thrombus verified between the preoperative and postoperative periods was expressed as a percentage. All statistical analyses were performed with SPSS Statistics 22 (IBM Corp., Armonk, New York, United States). A value of $p<0.05$ was considered statistically significant.

\section{Results}

The patients comprised 17 women (55\%) and 14 men (45\%), with a mean age of 46.7 years (range, 23-67 years). Among all GIAs, 15 (48.4\%) were identified as thrombotic aneurysms. The mean diameter of all GIAs was $28.7 \pm 1.5 \mathrm{~mm}$ (range, 25.9-31.9 mm). Most GIAs (93.5\%) were located in the anterior circulation; only two were located in the posterior circulation. In general, most clinical presentations of GIAs were attributed to a mass effect. Among all patients, 12 
Table 1 Patient and aneurysm characteristics

\begin{tabular}{|c|c|}
\hline \multicolumn{2}{|l|}{ Characteristics } \\
\hline Number of GIAs & 31 \\
\hline Patient sex, F/M & $17 / 14$ \\
\hline Patient age (y), mean (min, max) & $46.7(23,67)$ \\
\hline \multicolumn{2}{|l|}{ GIA location, number (\%) } \\
\hline Anterior circulation & $29(93.5 \%)$ \\
\hline Posterior circulation & $2(6.5 \%)$ \\
\hline \multicolumn{2}{|l|}{ Aneurysmal character, number (\%) } \\
\hline Thrombotic & $15(48.4 \%)$ \\
\hline Nonthrombotic & $16(51.6 \%)$ \\
\hline \multicolumn{2}{|l|}{ GIA diameter, mean $\pm S D(m m)$} \\
\hline DSA & $24.46 \pm 5.25$ \\
\hline 3D Slicer reconstruction & $28.67 \pm 1.48$ \\
\hline \multicolumn{2}{|l|}{ Volume, mean $\pm \mathrm{SD}\left(\mathrm{mm}^{3}\right)$} \\
\hline Thrombus & $3,590 \pm 1,003.7$ \\
\hline GIAs & $8292.6 \pm 1,175.1$ \\
\hline \multicolumn{2}{|l|}{ Clinical presentation, number (\%) } \\
\hline Vision defect & $12(38.7 \%)$ \\
\hline Headache & $8(25.8 \%)$ \\
\hline Ptosis & $2(6.5 \%)$ \\
\hline Limb weakness & $2(6.5 \%)$ \\
\hline Epilepsy & $2(6.5 \%)$ \\
\hline Hypomnesia & $1(3.2 \%)$ \\
\hline Negative & $4(12.9 \%)$ \\
\hline
\end{tabular}

Abbreviations: GIA, giant intracranial aneurysm; F, female; M, male; DSA, digital subtraction angiography.

presented with a vision defect, 8 with headache, 2 with ptosis, 2 with limb weakness, 2 with epilepsy, and 1 with hypomnesia. The remaining four patients had no obvious symptoms, and their GIAs were incidentally found. The patient and aneurysm characteristics are summarized in - Table 1.

The interobserver agreement was excellent for aneurysmal diameter and volume measurement by DSA or 3D Slicer reconstruction. The ICC for diameter by DSA, diameter by 3D Slicer, and volume by 3D Slicer was $0.98,0.87$, and 0.98 , respectively, and all corresponding $p$ values were $<0.001$ ( - Table 2). The mean diameter for all GIAs measured by DSA and 3D Slicer was $24.46 \pm 5.25$ and $28.66 \pm 1.48 \mathrm{~mm}$, respectively $(t=4.948, p<0.001)$. When only nonthrombotic GIAs were included, the mean diameter measured by DSA and 3D Slicer was $28.69 \pm 2.03$ and $28.97 \pm 1.79 \mathrm{~mm}$, respectively $(t=1.023, p=0.323$; - Table 3$)$. The mean aneurysmal volume was $8,292.6 \pm 1,175.1 \mathrm{~mm}^{3}$ and the mean thrombotic volume was $3,590.0 \pm 1,003.7 \mathrm{~mm}^{3}$. For thrombotic GIAs, the thrombus volume constituted $46.89 \pm 14.58 \%$ of the total aneurysm volume. The average time for all reconstruction procedures was $\sim 30$ minutes.
Table 2 The estimation of ICC between measurer

\begin{tabular}{|l|l|l|l|}
\hline & Type & ICC & $p$ \\
\hline Diameter & & & \\
\hline DSA & One-way random & 0.98 & $<0.001$ \\
\hline $\begin{array}{l}\text { 3D Slicer } \\
\text { reconstruction }\end{array}$ & Two-way random & 0.87 & $<0.001$ \\
\hline $\begin{array}{l}\text { Aneurysmal } \\
\text { volume }\end{array}$ & Two-way random & 0.98 & $<0.001$ \\
\hline
\end{tabular}

Abbreviations: ICC, intraclass correlation coefficient; DSA, digital subtraction angiography

Table 3 Paired $t$-test between two methods for diameter measurement

\begin{tabular}{|l|l|l|l|l|}
\hline & DSA (mm) & $\begin{array}{l}\text { 3D Slicer } \\
(\mathrm{mm})\end{array}$ & $t$ & $p$ \\
\hline All GIAs & $24.46 \pm 5.25$ & $28.66 \pm 1.48$ & 4.948 & $<0.001$ \\
\hline $\begin{array}{l}\text { GIAs } \\
\text { without } \\
\text { thrombus }\end{array}$ & $28.69 \pm 2.03$ & $28.97 \pm 1.79$ & 1.023 & 0.323 \\
\hline
\end{tabular}

Abbreviations: DSA, digital subtraction angiography; GIAs, giant intracranial aneurysms.

\section{Discussion}

Because they are tumorlike, GIAs often present with paroxysmal neurologic symptoms that are associated with a mass effect, ${ }^{2,4,14}$ and this may limit the ability to treat GIAs with endovascular intervention. In certain cases, open surgery may be the best choice. However, GIAs are always challenging to manage microsurgically. Therefore, meticulous imaging studies and individualized planning are required before surgery. Therapeutic planning for GIAs requires comprehensive images. DSA and CTA, both standard techniques, cannot reveal a thrombus in a GIA, ${ }^{8,13}$ which influences the accuracy of preoperative planning. The aneurysmal reconstruction with TOF and MSDE sequences by 3D Slicer in our series is a supplemental technique for surgical planning.

3D Slicer is a free open-source, extensible software application for medical image computing and segmentation. ${ }^{15}$ It can be used for quantitative assessment of the aneurysmal and thrombotic volume, which may be an independent risk factor for a poor outcome. ${ }^{16,17}$ The software allows us to not only measure the whole volume of the GIA but also split the volume into thrombotic and nonthrombotic portions (- Table $\mathbf{1}$; -Fig. 1, 2). Because they are compressed by peripheral brain tissue, progressive GIAs do not always present as an increase in size but instead by a change in volume. ${ }^{18,19}$ In such cases, the change in aneurysmal volume over time as measured by 3D Slicer can provide evidence for a high rupture risk, which is especially important for conservatively managed GIAs. In our series, all volumes were measured with the TOF sequence.

The TOF MRA imaging is often affected by artifacts resulting from slow and swirling blood flow in GIAs ${ }^{8,13,20}$ (-Fig. 2). MSDE was recently proposed for fast vessel wall imaging with accurate blood signal suppression and is mostly used for vessel 
wall imaging at the carotid bifurcation, ${ }^{12,21}$ where swirling flow is also present. Several reports have demonstrated the ability to use MSDE techniques for imaging of vessels in the intracranial region. ${ }^{22}$ In the present study, we could have reconstructed the thrombus with the MSDE sequence and integrated the data together with the circulatory portion reconstruction using the TOF sequence. However, the different parameters for the layer thickness between the two sequences could result in an inconsistent volume measurement; therefore, we only used the MSDE sequence as a reference for identification during the reconstructions in this series.

As a free open-source software accessible from the internet, 3D Slicer can run on a personal computer and does not require specialized equipment. Thus, it is easy to access at home or in the operating room. This feature is very important for neurosurgeons. Medical imaging, especially the MRI, is performed by radiologists. Although radiologists can offer neurosurgeons the best images with which to diagnose GIAs, they cannot consider the imaging findings from the neurosurgeon's intraoperative viewpoint. Additionally, traditional $2 \mathrm{D}$ images may be adequate for very experienced neurosurgeons, but a less experienced neurosurgeon may have difficulty extracting useful information. Therefore, 3D Slicer helps us to seize the initiative of processing image data to some extent.

The process of reconstruction is also a method with which to learn the anatomical relationship between GIAs and peripheral tissue before surgery, and the reconstructed model can be infused into a brain model and rotated at any angle (-Figs. 1, 2). From this aspect, 3D Slicer reconstruction can serve as a teaching tool for less experienced neurosurgeons. On the other hand, combined with the popular augmented reality (AR) technology, ${ }^{23}$ the reconstructed 3D images may optimize the workflow for surgical procedures by providing essential anatomical information that is entirely integrated into the surgical field. This is really worthy of a further exploration.

This study has some limitations. First, it may have been relatively time-consuming; the average time required for all reconstruction procedures was $\sim 30$ minutes. However, this time decreased as the learning curve progressed. Moreover, the time was dependent on the desired quality of the model. A coarser but adequately usable model for preoperative evaluation could be reconstructed even more rapidly. Second, the 3D Slicer is not approved by the Food and Drug Administration for certain clinical tasks and could only be used as a reference. However, this limitation is double-sided; the packages provide users with plenty of useful tools without any restrictions, as in commercial software. Third, no aneurysms of $<25 \mathrm{~mm}$ in diameter were included in our series, and the sample size (31 GIAs) was small.

\section{Conclusion}

In conclusion, GIAs are challenging to manage microsurgically, and meticulous imaging studies and individualized surgical planning are required before surgery. The MSDE sequence brings diagnostic benefit as comparison to other
MRI sequences. GIA reconstruction with 3D Slicer is a lowcost, dependable, and useful supplemental technique for surgical planning.

\section{Conflict of Interest}

None declared.

\section{References}

1 Wiebers DO, Whisnant JP, Huston J IIIInternational Study of Unruptured Intracranial Aneurysms Investigators. , et al; . Unruptured intracranial aneurysms: natural history, clinical outcome, and risks of surgical and endovascular treatment. Lancet 2003; 362(9378):103-110

2 Choi IS, David C. Giant intracranial aneurysms: development, clinical presentation and treatment. Eur J Radiol 2003;46(03): 178-194

3 Vorkapic P, Czech T, Pendl G, Oztürk E, Horaczek A. Clinicoradiological spectrum of giant intracranial aneurysms. Neurosurg Rev 1991;14(04):271-274

4 Hosobuchi Y. Direct surgical treatment of giant intracranial aneurysms. J Neurosurg 1979;51(06):743-756

5 Sharma BS, Gupta A, Ahmad FU, Suri A, Mehta VS. Surgical management of giant intracranial aneurysms. Clin Neurol Neurosurg 2008;110(07):674-681

6 Sughrue ME, Saloner D, Rayz VL, Lawton MT. Giant intracranial aneurysms: evolution of management in a contemporary surgical series. Neurosurgery 2011;69(06):1261-1270, discussion 1270-1271

7 Teng MM, Nasir Qadri SM, Luo CB, Lirng JF, Chen SS, Chang CY. MR imaging of giant intracranial aneurysm. J Clin Neurosci 2003;10 (04):460-464

8 Tollard E, Perot G, Clavier E, Gerardin E. Imaging of giant cerebral aneurysms. Neurochirurgie 2015;61(06):378-384

9 Li MH, Li YD, Tan HQ et al. Contrast-free MRA at 3.0 T for the detection of intracranial aneurysms. Neurology 2011;77(07): 667-676

10 Li MH, Cheng YS, Li YD, et al. Large-cohort comparison between three-dimensional time-of-flight magnetic resonance and rotational digital subtraction angiographies in intracranial aneurysm detection. Stroke 2009;40(09):3127-3129

11 Zhu C, Graves MJ, Yuan J, Sadat U, Gillard JH, Patterson AJ. Optimization of improved motion-sensitized driven-equilibrium (iMSDE) blood suppression for carotid artery wall imaging. J Cardiovasc Magn Reson 2014;16:61

12 Wang J, Yarnykh VL, Yuan C. Enhanced image quality in blackblood MRI using the improved motion-sensitized driven-equilibrium (iMSDE) sequence. J Magn Reson Imaging 2010;31(05): 1256-1263

13 Schaller B, Lyrer P. Focal neurological deficits following spontaneous thrombosis of unruptured giant aneurysms. Eur Neurol 2002;47(03):175-182

14 Park W, Chung J, Ahn JS, Park JC, Kwun BD. Treatment of large and giant middle cerebral artery aneurysms: risk factors for unfavorable outcomes. World Neurosurg 2017;102:301-312

15 Fedorov A, Beichel R, Kalpathy-Cramer J, et al. 3D Slicer as an image computing platform for the Quantitative Imaging Network. Magn Reson Imaging 2012;30(09):1323-1341

16 Sadato A, Hayakawa M, Tanaka T, Hirose Y. Comparison of cerebral aneurysm volumes as determined by digitally measured 3D rotational angiography and approximation from three diameters. Interv Neuroradiol 2011;17(02):154-158

17 Dengler J, Maldaner N, Bijlenga PGiant Intracranial Aneurysm Study Group. , et al; . Quantifying unruptured giant intracranial aneurysms by measuring diameter and volume: a comparative analysis of 69 cases. Acta Neurochir (Wien) 2015;157(03): 361-368, discussion 368 
18 van Keulen JW, van Prehn J, Prokop M, Moll FL, van Herwaarden JA. Potential value of aneurysm sac volume measurements in addition to diameter measurements after endovascular aneurysm repair. J Endovasc Ther 2009;16(04):506-513

19 Wever JJ, Blankensteijn JD, Mali WP Th M, Eikelboom BC. Maximal aneurysm diameter follow-up is inadequate after endovascular abdominal aortic aneurysm repair. Eur J Vasc Endovasc Surg 2000; 20(02):177-182

20 Takhtani D, Dundamadappa S, Puri AS, Wakhloo A. Flow artifact in the anterior communicating artery resembling aneurysm on the time of flight MR angiogram. Acta Radiol 2014;55(10):1253-1257
21 Obara M, Van Cauteren M, Honda M, Imai Y, Kuroda K. Assessment of improved motion-sensitized driven equilibrium (iMSDE) for multi-contrast vessel wall screening. Magn Reson Med Sci 2014; 13(02):139-144

22 Obara M, Kuroda K, Wang J, et al. Comparison between two types of improved motion-sensitized driven-equilibrium (iMSDE) for intracranial black-blood imaging at 3.0 tesla. J Magn Reson Imaging 2014;40(04):824-831

23 Karmonik C, Elias SN, Zhang JY, et al. augmented reality with virtual cerebral aneurysms: a feasibility study. World Neurosurg 2018;119:e617-e622 